\title{
Carcinoma sebáceo de la glándula parótida*
}

\author{
Drs. IGNACIO GOÑI E. ${ }^{1}$, PABLO MARIÁNGEL P. ${ }^{1}$, ANTONIETA SOLAR G. ${ }^{2}$, PELAYO BESA DE C. ${ }^{3}$
}

\author{
Departamento de Cirugía Oncológica y Maxilofacial. \\ Departamento de Anatomía Patológica. \\ Departamento de Radio-Oncología. \\ Pontificia Universidad Católica de Chile. \\ Santiago, Chile.
}

Abstract

\section{Sebaceous carcinoma of the parotid gland}

Introduction: Sebaceous carcinoma of the parotid gland is an extremely rare neoplasm, whose treatment is complex by the possible consequences that may occur due to its location. Material and Methods: We report a case of a 54 year old patient with a left parotid tumor without facial nerve dysfunction. The imaging study reports the presence of a left parotid tumor of $2.3 \mathrm{~cm}$, without lymphadenopathy. Results: We performed a left partial parotidectomy whose final pathological diagnosis was a sebaceous carcinoma of the parotid gland.

Key words: Parotid carcinoma, sebaceous carcinoma.

\section{Resumen}

Introducción: El carcinoma sebáceo de la glándula parótida es una neoplasia extremadamente infrecuente, cuyo manejo es complejo por las posibles secuelas que pueden suceder dada su localización. Material y Método: Se reporta el caso de una paciente de 54 años con un tumor parotídeo izquierdo de larga data sin compromiso de la función del VII par. Resultados: El estudio por imágenes informó la presencia de un tumor parotídeo izquierdo de $2,2 \mathrm{~cm}$, sin linfoadenopatías regionales. Se realizó una parotidectomía parcial izquierda cuyo diagnóstico patológico definitivo fue un carcinoma sebáceo de glándula parótida.

Palabra clave: Carcinoma parotídeo, carcinoma sebáceo.

\section{Introducción}

Los carcinomas salivales con diferenciación sebácea son extremadamente raros, siendo la glándula parótida la localización más común, con alrededor de 30 casos reportados en la literatura.
El objetivo de este trabajo es reportar un caso de un carcinoma sebáceo de glándula parótida tratado en nuestra institución, exponer los hallazgos clínicos e imagenológicos y finalmente, discutir el resultado anatomopatológico y el tratamiento efectuado.

*Recibido el 7 de mayo de 2012 y aceptado para publicación el 2 de junio de 2012.

Los autores no refieren conflictos de interés.

Correspondencia: Dr. Ignacio Goñi E.

Marcoleta 350, Santiago, Chile.

igoni@med.puc.cl 


\section{Caso clínico}

Paciente de sexo femenino, 53 años, hipertensa en tratamiento. Historia de aumento de volumen preauricular izquierdo, de varios años de evolución, indoloro, sin evidencia clínica de compromiso del VII par. Por la aparición de eritema local en relación al tumor consultó en el Equipo de Cirugía Oncológica de la P. Universidad Católica de Chile. Se solicitó tomografía de cuello que mostró la presencia de un proceso expansivo sólido del segmento apical del lóbulo superficial de la glándula parótida izquierda, que como primera posibilidad considera un adenoma pleomorfo. Se complementa el estudio con una resonancia magnética de cuello que informó un proceso expansivo bien delimitado anterosuperior superficial de la glándula parótida izquierda. Sin adenopatías (Figura 1). Por estos hallazgos se indicó resección quirúrgica de la lesión. En el intraoperatorio se visualizó un tumor del lóbulo superficial de la parótida, bien delimitado, pétreo, en estrecha relación con las ramas del VII par. Se tomó una biopsia incisional para determinar la extensión de la resección y la eventual necesidad de incluir en ella ramas del nervio facial. Esta biopsia informó tumor parotídeo compatible con carcinoma sebáceo $\mathrm{v} / \mathrm{s}$ carcinoma mucoepidermoide. Ante estos hallazgos se realizó una parotidectomía parcial, resecando un tumor de 2,3 $\mathrm{cm}$ y preservando las ramas del VII par. El tumor invadía aparentemente el arco zigomático, en su porción central, que también se extirpó. Evolucionó en el postoperatorio con paresia de la rama zigomaticotemporal del VII par. La biopsia definitiva informó un carcinoma sebáceo, bien diferenciado, de 2,3 $\times 1,9 \times 1,3 \mathrm{~cm}$. El tumor infiltra el tejido extraparenquimatoso y alcanza focalmente el borde quirúrgico. No se reconocen permeaciones vasculares ni infiltración perineural tumoral. Se realizó la tinción histoquímica de Oil Red $O$ que demostró ácidos grasos intracitoplasmáticos en las células claras. Tejido óseo sin evidencia de neoplasia. Se catalogó como pT3N0M0. Se realizó radioterapia adyuvante (50 Gy en 25 fracciones más bolus hasta completar $60 \mathrm{~Gy}$ ). A 12 meses, la paciente se encuentra sin evidencia de recidiva locorregional y con recuperación completa de la función del VII par.

\section{Discusión}

El carcinoma sebáceo es una neoplasia infrecuente pero agresiva, que provoca importante destrucción local y posee una alta capacidad metastásica. La localización más frecuente es la zona palpebral que involucra aproximadamente el $75 \%$ de los casos. La presencia de carcinomas sebáceos cuyo origen sea una glándula salival es extremadamente rara. Se han reportado sólo 28 casos de esta entidad hasta el año $2003^{1}$. El $70 \%$ se origina en la glándula parótida y con menor frecuencia en la glándula submandibular. Se caracterizan por ser tumores de crecimiento lento, indoloros, que raramente afectan el nervio facial. El pronóstico de los carcinomas sebáceos extraoculares es favorable, debido a que poseen una baja capacidad metastásica a diferencia de la localización palpebral. La edad de presentación es variable, reportándose desde los 19 hasta 93 años, siendo más frecuente en la tercera edad. La incidencia es similar entre hombres y mujeres ${ }^{2}$. Los factores de riesgo descritos son²:

1. Edad avanzada

2. Ascendencia asiática o sud-asiática

3. Sexo femenino

4. Antecedente de radioterapia de cabeza y cuello

5. Síndrome de Muir-Torre

6. Retinoblastoma familiar

Estos carcinomas pueden presentar características histológicas similares a un carcinoma mucoepidermoide/escamoso e incluso a un adenoma sebáceo, por lo que el uso de tinciones especiales (Oil-Red O, glicógeno, mucina) son indispensables para el diagnóstico específico. Los carcinomas sebáceos son neoplasias bien diferenciadas en su mayoría compuestas por nidos de células con citoplasma vacuolado o espumoso ${ }^{4}$. En nuestro paciente el estudio histopatológico informó la presencia de nidos de células neoplásicas claras, con vacuolización del citoplasma, de núcleos pleomórficos, rodeadas por estroma colagenoso, hialino, abundante (Figuras 2 y 3). La tinción del Oil Red $O$ fue positiva en el citoplasma de las células tumorales (Figura 4), confirmando la presencia de contenido. En tumores bien diferenciados, el diagnóstico entre carcinoma

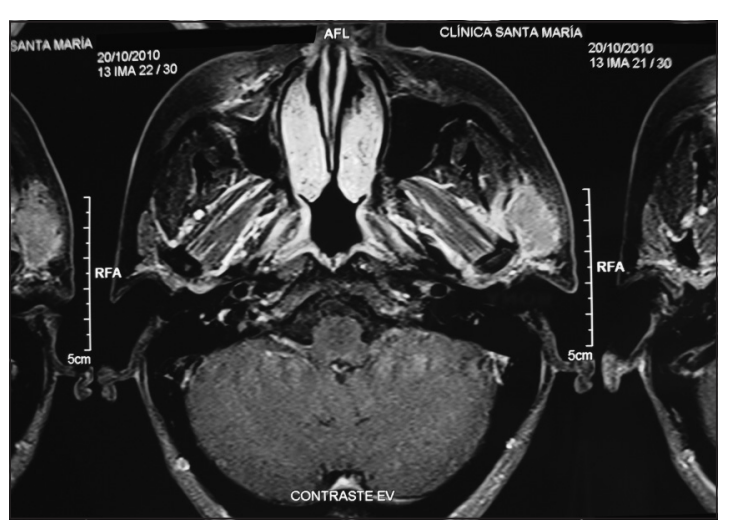

Figura 1. Proceso expansivo sólido, de 2,2 × 2 × 1,9 cm, localizado en la porción anterosuperior superficial de la glándula parótida izquierda. 


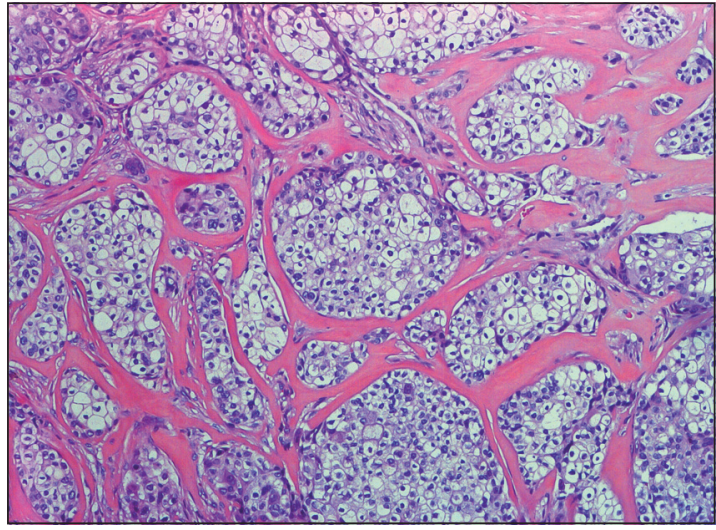

Figura 2. Se observan nidos pequeños y grandes, irregulares, así como la presencia de células aisladas en tejido glandular fibroso, que muestra hialinización prominente (HE 100x).

y adenoma puede ser complejo. La presencia de un patrón infiltrativo y desordenado es sugerente de un carcinoma, no obstante en casos difíciles, el estudio inmunohistoquímico (p53 $\geq 10 \%$, Ki67 $\geq 25 \%$, bcl-2 negativo o $\leq 10 \%$ ) apoyaría el diagnóstico de carcinoma ${ }^{5}$.

Dada la rareza de esta neoplasia, no hay tratamientos específicos. La parotidectomía parcial o total, con márgenes libres (10 $\mathrm{mm})$, asociado al uso de radioterapia adyuvante es el tratamiento de elección reportado en la literatura. La radioterapia exclusiva no se recomienda, sin embargo, no hay suficiente evidencia para apoyar esta afirmación ${ }^{3}$. Los criterios de conservación del nervio facial son similares a los utilizados con otras neoplasias parotídeas.

En nuestra paciente destaca una historia larga que es concordante con la forma de presentación habitual de este tumor. El enfoque diagnóstico y terapéutico fue similar al utilizado con cualquier masa parotídea. En este caso, se evidenció compromiso de uno de los márgenes quirúrgicos, razón por la que se indicó radioterapia adyuvante.

\section{Referencias}

1. Siriwardena BS, Tilakaratne WM, Rajapakshe RM. A case of sebaceous carcinoma of the parotid gland. J Oral Pathol Med. 2003;32:121-3.

2. Dasgupta T, Wilson LD,Yu JB. A retrospective review of 1349 cases of sebaceous carcinoma. Cancer 2009; 115:158-65. doi: 10.1002/cncr.23952.

3. Tryggvason G, Bayon R, PagedarNA. Epidemiology of

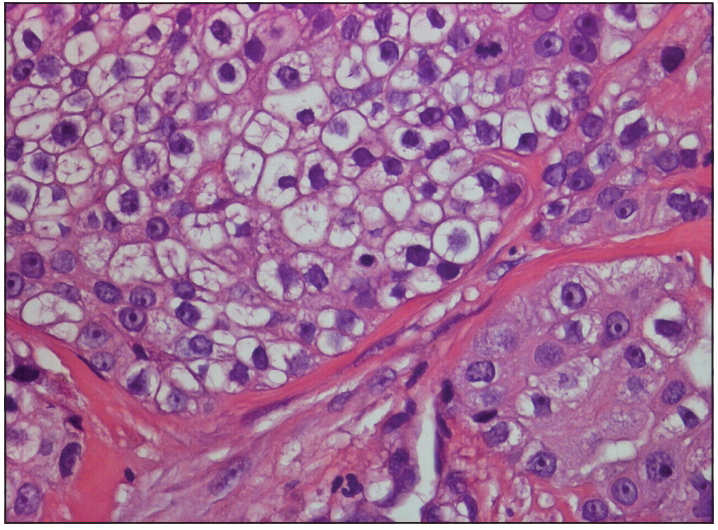

Figura 3. Con aumento mayor se observa células claras con vacuolización del citoplasma y otras con citoplasma eosinófilo. Los núcleos son moderadamente pleomórficos, redondeados, con cromatina fina e hipercromáticos, con nucléolo prominente. Se identifica una figura mitótica en la esquina superior derecha (HE 400x).

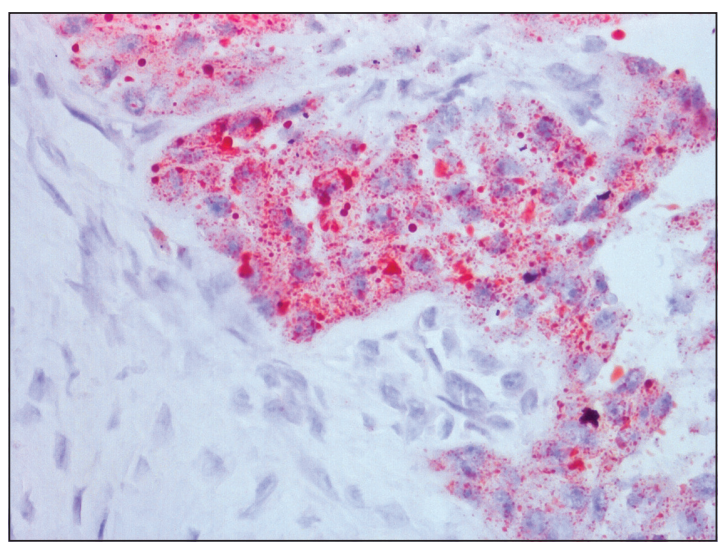

Figura 4. Tinción de Oil Red $\mathrm{O}$, que destaca en rojo el contenido sebáceo $(400 x)$.

sebaceous carcinoma of the head and neck: Implications for lymph node management. Head \& Neck 2012 doi: 10.1002/hed.22009.

4. Misago N, Toda S, Narisawa Y. Two histopathologic patterns of well-differentiated extraocular sebaceous carcinoma. Journal of Cutaneous Pathology 2011; 38:76774. doi: 10.1111/j.1600-0560.2011.01759.

5. Cabral ES, Auerbach A, Killian JK, Barrett TL, Cassarino DS. Distinction of benign sebaceous proliferations from sebaceous carcinomas by immunohistochemistry. Am J Dermatopathol. 2006;28:465. 